\title{
Complementary and Alternative Medicines Use during Pregnancy: A Systematic Review of Pregnant Women and Healthcare Professional Views and Experiences
}

\author{
Abdul Rouf Pallivalappila, ${ }^{1}$ Derek Stewart, ${ }^{2}$ Ashalatha Shetty, ${ }^{3}$ \\ Binita Pande, ${ }^{3}$ and James S. McLay ${ }^{1}$ \\ ${ }^{1}$ Division of Applied Health Sciences, Medical and Dental School, University of Aberdeen, Aberdeen AB25 2ZG, UK \\ ${ }^{2}$ School of Pharmacy and Life Sciences, Robert Gordon University, Aberdeen AB10 7QB, UK \\ ${ }^{3}$ Aberdeen Maternity Hospital, Aberdeen AB25 2ZL, UK \\ Correspondence should be addressed to James S. McLay; j.mclay@abdn.ac.uk
}

Received 11 March 2013; Revised 12 June 2013; Accepted 21 August 2013

Academic Editor: Thomas Ostermann

Copyright (C) 2013 Abdul Rouf Pallivalappila et al. This is an open access article distributed under the Creative Commons Attribution License, which permits unrestricted use, distribution, and reproduction in any medium, provided the original work is properly cited.

\begin{abstract}
Aims. To undertake a systematic review of the recent (2008-2013) primary literature, describing views and experiences of CAM use during pregnancy by women and healthcare professionals. Method. Medline, Cumulative Index to Nursing and Allied Health Literature, Cochrane Database of Systematic Review Library and Allied, and Complementary Medicine Database were searched. Studies reporting systemic CAM products (homeopathic preparations, herbal medicines, Vitamins and minerals, homeopathy, and special diets) alone or in combination with other nonsystemic CAM modalities (e.g., acupuncture) were included. Results. Database searches retrieved 2,549 citations. Removal of duplicates followed by review of titles and abstracts yielded 32 relevant studies. Twenty-two reported the perspectives of women and their CAM use during pregnancy, while 10 focused on healthcare professionals. The majority of studies had significant flaws in study design and reporting, including a lack of appropriate definitions of CAM and associated modalities, absence of detailed checklists provided to participants, the use of convenience sampling, and a general lack of scientific robustness in terms of data validity and reliability. Conclusion. To permit generalisability of study findings, there is an urgent need to expand the evidence base assessing CAMs use during pregnancy using appropriately designed studies.
\end{abstract}

\section{Introduction}

The World Health Organisation defines "complementary and alternative medicine" (CAM) as a "broad set of health care practices that are not part of that country's own tradition and are not integrated into the dominant health care system" [1]. However the scope of CAM is broad and various including therapies such as acupuncture, aromatherapy, herbal, and homeopathic medicines [2]. Whist acknowledging limitations of the published literature (varying definitions of CAM, bias, and confounding), studies undertaken throughout the world have reported women to be large consumers of CAM with prevalence figures of with $56-88 \%$ of UK women [3], over $50 \%$ of middle aged and older Australian women [4], and over $90 \%$ of menopausal Canadian women [5]. Unlike conventional licensed medicines, few CAM approaches to healthcare are supported by robust efficacy, effectiveness, or safety data [6,7], which raises potential concerns about the use of CAM, particularly in high risk patients such as pregnant women where teratogenicity is a concern [8].

Several limited literature reviews have focused on the use of CAMs by pregnant women [9-11] and recommendations for use by health professionals during pregnancy $[9,12]$. While these reviews have concentrated on the findings of relevant CAM-based studies, they have placed less emphasis on critically appraising the core elements of study design and reporting. Limited reviews of CAM recommendations made by healthcare professionals report that CAM approaches, particularly herbal therapy, chiropractic, acupuncture/acupressure, massage, homoeopathy, and 
aromatherapy are commonly recommended and used in the maternity setting.

We report a systematic review of the primary literature published over the last five years (2008-2012) focusing on the views and experiences of CAM use during pregnancy by women and healthcare professionals, with emphasis on study design and limitations of findings.

\section{Method}

2.1. Search Strategy. A systematic review protocol was prepared as per standard guidelines $[13,14]$. The databases Medline, Cumulative Index to Nursing and Allied Health Literature (CINAHL), Cochrane Database of Systematic Review (CDSR) library, and Allied and Complementary Medicine Database (AMED) were searched from January 2008 to December 2012 inclusive, using the search terms (as keywords, title, and abstract) of pregnancy, complementary, alternative, herbal, homeopathic, vitaminmidwife, obstetrician, doctor, physician, woman, patient, views, opinions, experiences, and prevalence. Wildcard symbols, truncation, combinations of search terms using Boolean operators, and alterative spellings were used. Included studies reported either women and/or healthcare professionals' perspectives of prevalence and outcomes of CAM use. Studies reporting systemic CAM products (homeopathic preparations, herbal medicines, vitamins and minerals, homeopathy, and special diets) either alone or in combination with other nonsystemic CAM modalities (e.g., acupuncture) were included. The reference lists from identified papers were scanned for other relevant studies. The search was limited to English language articles. Studies published only as abstracts, letters, or conference proceedings were excluded.

Initial screening of titles was carried out to identify potentially relevant studies, followed by screening of abstracts and then by full paper review. Fifty titles and abstracts were independently evaluated by two reviewers for consistency of inclusion/exclusion.

2.2. Study Review and Data Extraction. Independent quality assessments were conducted by two independent reviewers utilising standard criteria for critical appraisal $[15,16]$ (Table 1).

Data extracted were; country of study, CAM definitions and scope, sample size and response rate, prevalence of use, perceived effectiveness and safety, and predictors of CAM use. Due to lack of study homogeneity, a narrative synthesis of the results was conducted.

\section{Results}

Database searches retrieved 2,549 citations. Removal of duplicates followed by review of titles and abstracts yielded 32 relevant studies. The PRISMA flowchart (Figure 1) illustrates the number of titles, abstracts, and full papers excluded. Twenty-two studies reported the perspectives of women and their CAM use during pregnancy, while 10 focused on healthcare professionals.
TABLE 1: Criteria for critical appraisal.

(1) Is a definition of CAMs/herbals given?

(2) Is a description of CAMs/herbals checklist given?

(3) Is the information about the ethics given (approved/not required)?

(4) Are study inclusion and exclusion criteria fully described?

(5) Is the sample size fully justified?

(6) Is development of questionnaire (validity, reliability, and piloting) fully described?

(7) Are study strengths and limitations fully discussed?

(8) Is generalisability fully discussed?

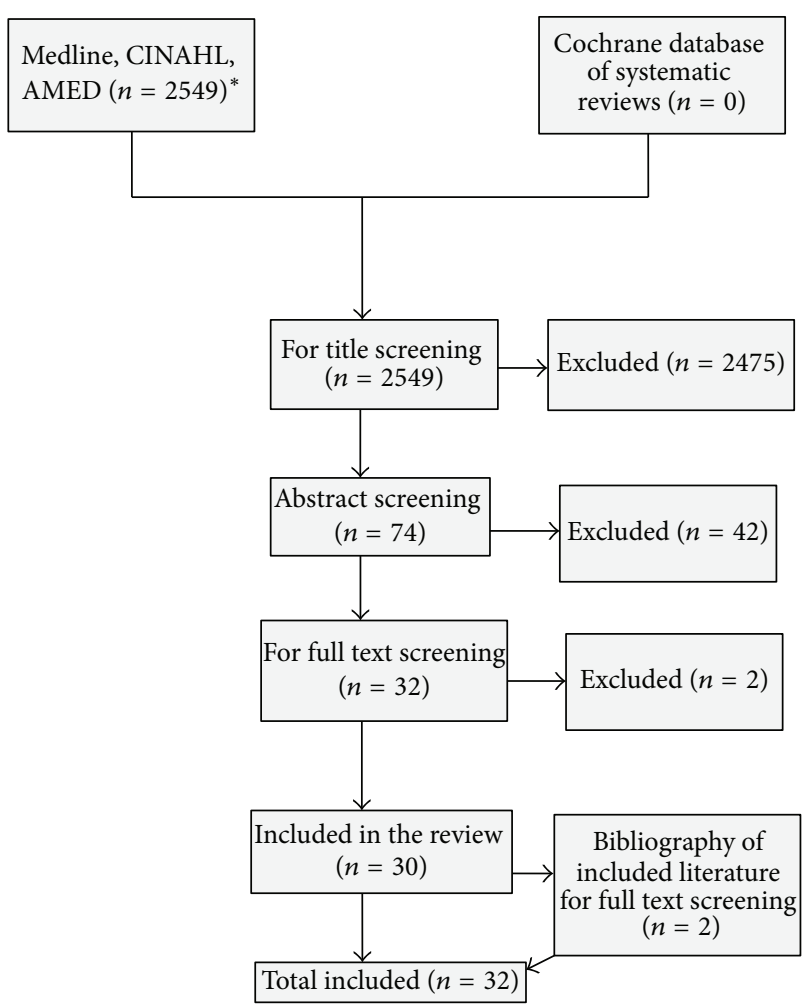

FIGURE 1: PRISMA flowchart, ${ }^{*}$ after removal of duplicates.

Included studies were conducted in Europe $(n=13)$, the Americas $(n=8)$, Asia $(n=6)$, Australia and Oceania $(n=$ $4)$, and Africa $(n=1)$. Data were collected by questionnaire $(n=21)$ or structured interview $(n=11)$. Findings of the critical appraisal are reported in Table 2 and Figure 2. Summaries of study details are provided in Table 3 (women) and Table 4 (health professionals). Data should be interpreted with caution due to inconsistency and lack of CAM and CAM related definitions, limitations of study design, and a paucity of outcomes data.

3.1. Studies Assessing Experience and Views of Women ( $n=$ 22). Eleven of the 22 studies reported on more than one CAM modality, nine focused on herbals only and two studies reported herbals, and vitamins. 


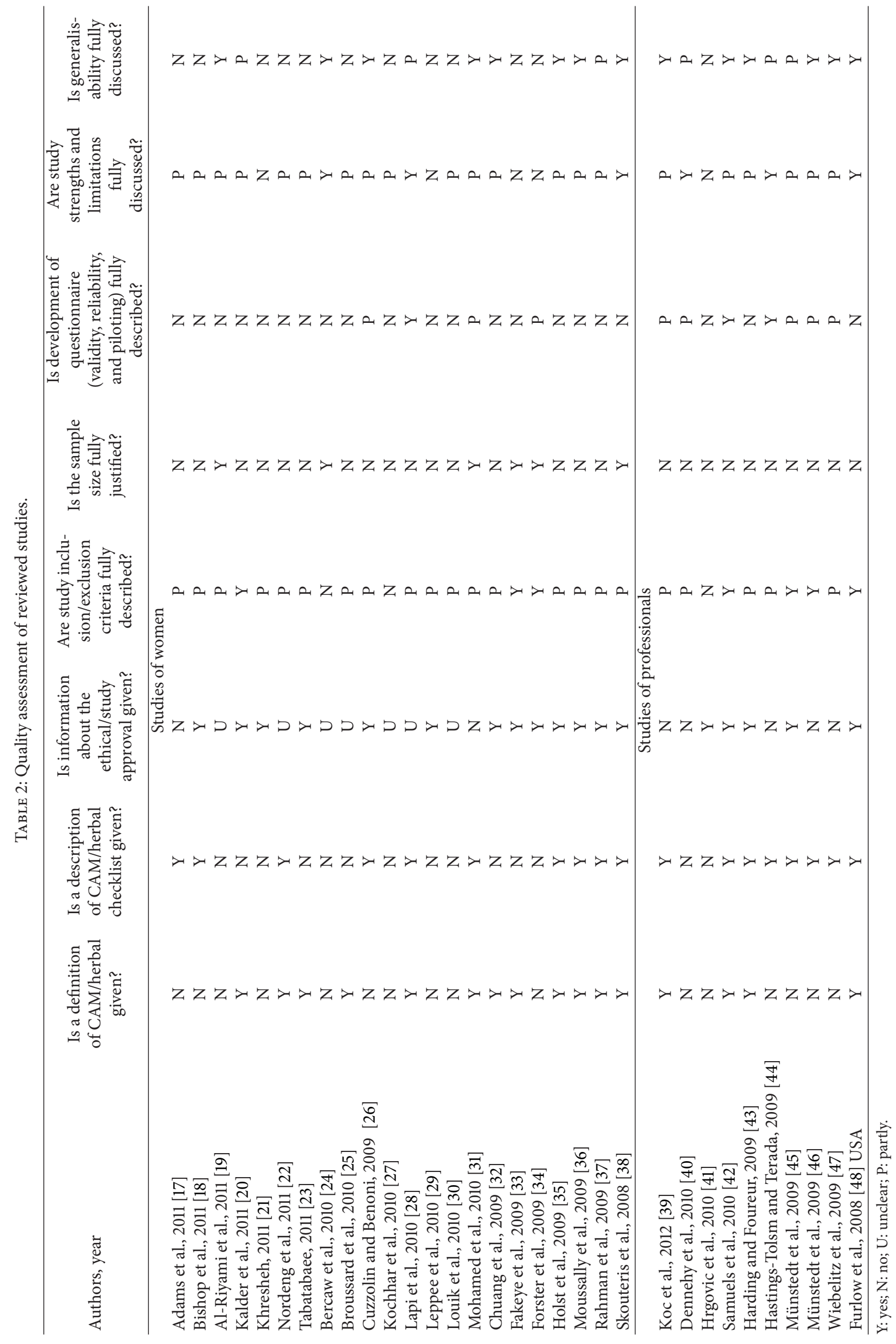




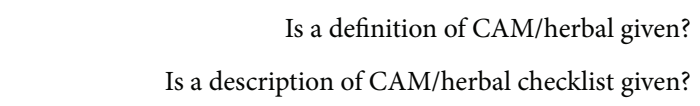

Is information about the ethical review/study approval given?

Are study inclusion and exclusion fully described?

Is the sample size fully justified?

Is development of questionnaire (validity, reliability, piloting) fully described?

Are study strengths and limitations fully discussed?

Is generalisability fully discussed?
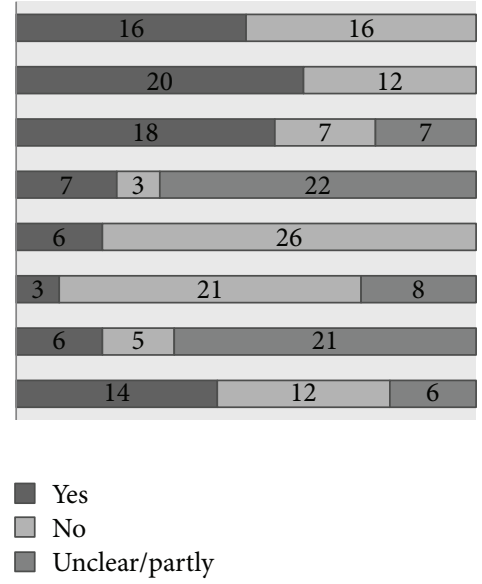

FIGURE 2: Stacked bar chart representing quality assessment of reviewed studies $(n=32)$.

3.1.1. Definitions and Scope. Of the 11 studies reporting data for more than one CAM modality, only four [20, 28, 31, 38 ] defined the term "CAM". Five of these 11 studies [17, $18,28,31,38]$ provided a detailed and specific checklist of CAM modalities and products to research participants. However, the content and specificity of these checklists were highly variable, precluding direct interstudy comparisons. For example, Adams et al. [17] listed

"vitamins or minerals, yoga/meditation, herbal medicines, aromatherapy oils, and Chinese medicines"

while Bishop et al. [18] included

"treatments, pills, medicines, ointments, homeopathic medicines, herbal medicines, supplements, drinks, and herbal teas".

No information on study CAM checklists was provided in the remaining six studies [19-21, 24, 27, 30].

Of the nine studies assessing only herbals use, eight provided a definition of herbal medicine $[22,23,25,32,33,35-$ 37]. However, no consistent definition was used. For example, Tabatabaee [23] defined "herbal drugs" as

"all types of products (oral and dermatological) that were manufactured from herbs or contained herbs as the major component"

while Holst et al. [35] defined "herbal remedies" as

"any kind of product such as a tablet, a mixture, an ointment or herbal teas which are produced from plants and used to acquire better health".

Only five of these nine studies detailed the specific checklist of herbals provided to research participants [22, 26, 35-37]. The content of these checklists varied greatly listing between nine and 40 products, with a free text option to permit participants to add unlisted herbal products.
3.1.2. Study Design. Only two of the 22 studies assessing the experiences and views of women $[18,19]$ used the ideal prospective, longitudinal study design, collecting data at several time points during pregnancy and following delivery. However, while Al-Riyami et al. [19] collected data 3 months prior to pregnancy and at each trimester, the study reported by Bishop et al. [18] had a 20-year gap between data collection (1991-1992) and study publication in 2011 raising significant concerns around current validity of data.

The remaining 20 studies reported collection of retrospective data, thus introducing potential recall bias with implications for data validity and reliability. For example, participants in the study reported by Moussally et al. were required to recall herbal products used during pregnancy up to eight years previously [36].

Sample size varied greatly amongst these 22 studies, with participant numbers varying between 139 and 14,541. Only six studies [19, 24, 31, 33, 34, 37] described a sample size calculation detailing estimates of data precision, confidence intervals, and likely response rates; calculated sample sizes varied from 122 to 600 .

The majority of studies used a convenience approach for participant sampling and recruitment, which reduces external validity of the findings. Response rates for questionnaire studies ranged from 39.0 to $99.0 \%$. Two studies attempted to demonstrate homogeneity between respondents and nonrespondents [22, 36]. However, Moussally et al. [36] reported similarity of respondents and nonrespondents in terms of maternal age, gestational age, rate of hospitalisations, pre-natal visits, and comorbidities while Nordeng et al. [22] reported the similarity between respondents and the general Norwegian postnatal population in terms of frequencies of age, parity, and marital status.

3.1.3. Study Findings. Notwithstanding limitations of study design and reporting, it is evident that a significant proportion of women use CAM during pregnancy (see Table 3). Prevalence rates ranged from $5.8 \%$ of 4,866 respondents in USA taking herbal or natural treatments [30] to $74.2 \%$ of 461 Hispanic women in the USA taking herbals [27]. Only nine 
TABLE 3: Data extraction relating to studies of women $(n=22)$.

\begin{tabular}{|c|c|c|c|c|c|}
\hline $\begin{array}{l}\text { Authors, year, } \\
\text { country }\end{array}$ & CAM studied & $\begin{array}{l}\text { Stage of } \\
\text { pregnancy at } \\
\text { point of data } \\
\text { collection }\end{array}$ & $\begin{array}{l}\text { Mode of data } \\
\text { collection }\end{array}$ & $\begin{array}{l}\text { Response rate } \\
\quad(\mathrm{RR}, \%)\end{array}$ & Prevalence in pregnancy \\
\hline $\begin{array}{l}\text { Adams et al., } \\
2011[17] \\
\text { Australia }\end{array}$ & CAM & Pregnant & Questionnaire & $\begin{array}{c}897 \text { ( } \mathrm{RR} \text { not } \\
\text { given) }\end{array}$ & $\begin{array}{l}81 \% \text { used vitamins and minerals, } \\
16 \% \text { aromatherapy, and } 15 \% \text { herbal } \\
\text { medicines. } \\
\text { All significantly higher than } \\
\text { nonpregnant women }(P<0.001) \text {. } \\
\text { No data on stage of pregnancy. }\end{array}$ \\
\hline $\begin{array}{l}\text { Bishop et al., } \\
2011 \text { [18], UK }\end{array}$ & CAM & $\begin{array}{c}\text { Antenatal } \\
\text { (weeks } 8,12 \text {, } \\
18,32)\end{array}$ & Questionnaire & $\begin{array}{l}\text { Total response } \\
\text { of } 14,115 / 14,541 \\
\text { (97.1) but varied } \\
\text { at each point of } \\
\text { data collection }\end{array}$ & $\begin{array}{l}26.7 \% \text { used a CAM at least once. } \\
\text { Most commonly herbal teas }(17.7 \%) \text {, } \\
\text { homeopathy }(14.4 \%) \text {, and herbal } \\
\text { medicines }(5.8 \%) \text {. } \\
\text { Prevalence increased each trimester } \\
(6 \%, 12.4 \%, 26.5 \% \text {, no } P \text { values). }\end{array}$ \\
\hline $\begin{array}{l}\text { Al-Riyami et al., } \\
2011 \text { [19], Oman }\end{array}$ & CAM & $\begin{array}{l}\text { Antenatal } \\
\text { (prenatal, } \\
\text { trimesters } 1 \text {, } \\
2,3 \text { ) }\end{array}$ & Interview & $\begin{array}{l}139 \text { but varied at } \\
\text { each data } \\
\text { collection point }\end{array}$ & $\begin{array}{l}\text { Compared to prenatal, vitamin used } \\
\text { increased from } 12 \% \text { to } 84-95 \% \\
\text { across three semesters }(P<0.001) \text {, } \\
\text { herbal medicines from } 7 \% \text { to } \\
\text { 16-19\% }(P<0.05) \text {. }\end{array}$ \\
\hline $\begin{array}{l}\text { Kalder et al., } \\
2011[20], \\
\text { Germany }\end{array}$ & CAM & Postnatal & Questionnaire & $205 / 475(43.2)$ & $\begin{array}{l}50.7 \% \text { used CAM (including CAM } \\
\text { practices). Homeopathy (18.5\%), } \\
\text { aromatherapy ( } 4.4 \%) \text {. } \\
\text { No data on stage of pregnancy. }\end{array}$ \\
\hline $\begin{array}{l}\text { Khresheh, } 2011 \\
\text { [21], Jordan }\end{array}$ & $\begin{array}{l}\text { CAM used in } \\
\text { treatment of } \\
\text { nausea and } \\
\text { vomiting }\end{array}$ & $\begin{array}{l}\text { Currently } \\
\text { pregnant or } \\
\text { at least one } \\
\text { full term } \\
\text { pregnancy }\end{array}$ & Questionnaire & $235 / 290(81.0)$ & $\begin{array}{l}9.4 \% \text { used herbal medicines. } \\
\text { No data on stage of pregnancy. }\end{array}$ \\
\hline $\begin{array}{l}\text { Nordeng et al., } \\
2011[22], \\
\text { Norway }\end{array}$ & Herbal & $\begin{array}{c}\text { Postnatal, } \\
\text { within } 5 \text { days }\end{array}$ & Interview & 600 & $\begin{array}{l}39.7 \% \text { used herbal medicines, } 4.3 \% \\
\text { homeopathy. } \\
\text { No data on stage of pregnancy. }\end{array}$ \\
\hline $\begin{array}{l}\text { Tabatabaee, } 2011 \\
\text { [23], Iran }\end{array}$ & Herbal & $\begin{array}{l}\text { Postnatal, } 2 \\
\text { days }\end{array}$ & Interview & 513 & $\begin{array}{l}30.8 \% \text { used herbal medicines. } \\
\text { Tended to be used more in first } \\
\text { trimester (no } P \text { values). }\end{array}$ \\
\hline $\begin{array}{l}\text { Bercaw et al., } \\
2010 \text { [24], USA }\end{array}$ & $\begin{array}{l}\text { Herbal and } \\
\text { vitamins }\end{array}$ & $\begin{array}{l}\text { Postnatal, } \\
\text { immediately }\end{array}$ & Questionnaire & $\begin{array}{l}485 \text { Hispanic } \\
\text { women (RR not } \\
\text { given) }\end{array}$ & $\begin{array}{l}19 \% \text { used herbal medicines, } 47 \% \\
\text { vitamins. } \\
\text { No data on stage of pregnancy. }\end{array}$ \\
\hline $\begin{array}{l}\text { Broussard et al., } \\
2010 \text { [25], USA }\end{array}$ & Herbal & $\begin{array}{l}\text { Postnatal, } 6 \\
\text { weeks-2 } \\
\text { years }\end{array}$ & Interview & 4239 & $\begin{array}{l}9.4 \% \text { used herbal medicines, the } \\
\text { highest for first trimester ( } 6.4 \% \text { v } 5.1 \\
\text { and } 5.2 \%) \text { (no } P \text { values). }\end{array}$ \\
\hline $\begin{array}{l}\text { Cuzzolin and } \\
\text { Benoni, } 2009 \\
{[26], \text { Italy }}\end{array}$ & Herbal & $\begin{array}{c}\text { Postnatal, } \\
\text { within } 3 \text { days }\end{array}$ & Interview & 392 & $\begin{array}{l}27.8 \% \text { used one or more herbal } \\
\text { medicines. Prevalence increased } \\
\text { slightly with trimester (no details or } \\
P \text { values). }\end{array}$ \\
\hline $\begin{array}{l}\text { Kochhar et al., } \\
2010 \text { [27], USA }\end{array}$ & Herbal & Not stated & Questionnaire & $\begin{array}{c}461 \text { Hispanic } \\
\text { women (RR not } \\
\text { given) }\end{array}$ & $\begin{array}{l}74.2 \% \text { used herbal medicines. } \\
\text { No data on stage of pregnancy. }\end{array}$ \\
\hline
\end{tabular}


TABLE 3: Continued.

\begin{tabular}{|c|c|c|c|c|c|}
\hline $\begin{array}{l}\text { Authors, year, } \\
\text { country }\end{array}$ & CAM studied & $\begin{array}{l}\text { Stage of } \\
\text { pregnancy at } \\
\text { point of data } \\
\text { collection }\end{array}$ & $\begin{array}{l}\text { Mode of data } \\
\text { collection }\end{array}$ & $\begin{array}{l}\text { Response rate } \\
\quad(\mathrm{RR}, \%)\end{array}$ & Prevalence in pregnancy \\
\hline $\begin{array}{l}\text { Lapi et al., } 2010 \\
\text { [28], Italy }\end{array}$ & CAM & $\begin{array}{l}\text { Antenatal, } \\
\text { trimester } 3\end{array}$ & Interview & 150 & $\begin{array}{l}48 \% \text { used CAM. } \\
\text { No data on stage of pregnancy. }\end{array}$ \\
\hline $\begin{array}{l}\text { Leppee et al., } \\
2010 \text { [29], } \\
\text { Crotia, Serbia }\end{array}$ & $\begin{array}{l}\text { Vitamins, } \\
\text { minerals and } \\
\quad \text { iron }\end{array}$ & Antenatal & Interview & 6,992 & $\begin{array}{l}56.6 \% \text { used vitamins and minerals } \\
\text { in Croatia, } 20.3 \% \text { in Serbia. } \\
\text { Prevalence increased with trimester } \\
\text { (no details or } P \text { values). }\end{array}$ \\
\hline $\begin{array}{l}\text { Louik et al., } 2010 \\
\text { [30], USA }\end{array}$ & $\begin{array}{l}\text { Herbal or } \\
\text { natural } \\
\text { treatments }\end{array}$ & $\begin{array}{l}\text { Postnatal, } \\
\text { within } 6 \\
\text { months }\end{array}$ & Interview & 4,866 & $\begin{array}{l}5.8 \% \text { used herbal or natural } \\
\text { treatments. } \\
\text { No data on stage of pregnancy. }\end{array}$ \\
\hline $\begin{array}{l}\text { Mohamed et al., } \\
2010 \text { [31], Qatar }\end{array}$ & CAM & Antenatal & Interview & 393 & $\begin{array}{l}67.5 \% \text { used CAM in first trimester, } \\
37.7 \% \text { in second, and } 28.9 \% \text { in third } \\
\text { (no } P \text { values). Mainly herbal } \\
\text { medicines and food supplements. }\end{array}$ \\
\hline $\begin{array}{l}\text { Chuang et al., } \\
2009[32], \\
\text { Taiwan }\end{array}$ & $\begin{array}{l}\text { Chinese } \\
\text { herbal }\end{array}$ & Postnatal & Interview & 21,248 & $\begin{array}{l}33.6 \% \text { used Chinese herbal } \\
\text { medicines. } \\
\text { No data on stage of pregnancy. }\end{array}$ \\
\hline $\begin{array}{l}\text { Fakeye et al., } \\
2009 \text { [33], } \\
\text { Nigeria }\end{array}$ & Herbal & Antenatal & Questionnaire & $560 / 600(99.0)$ & $\begin{array}{l}67.5 \% \text { used herbal medicines. } \\
\text { No data on stage of pregnancy. }\end{array}$ \\
\hline $\begin{array}{l}\text { Forster et al., } \\
2009[34] \\
\text { Australia }\end{array}$ & $\begin{array}{c}\text { Folic acid and } \\
\text { other vitamin } \\
\text { supplements }\end{array}$ & $\begin{array}{l}\text { Antenatal, } \\
\sim 38 \text { weeks }\end{array}$ & Questionnaire & $588 / 705(83.4)$ & $\begin{array}{l}91 \% \text { took at least one vitamin } \\
\text { supplement (mainly folic acid) } \\
\text { during pregnancy. } \\
\text { Almost all commenced during } \\
\text { trimester } 1 \text {. }\end{array}$ \\
\hline $\begin{array}{l}\text { Holst et al., } 2009 \\
\text { [35], UK }\end{array}$ & Herbal & $\begin{array}{l}\text { Antenatal, } \\
\text { more than } 20 \\
\text { weeks }\end{array}$ & Questionnaire & $578 / 1,037(55.7)$ & $\begin{array}{l}57.8 \% \text { used herbal medicines. } \\
\text { No data on stage of pregnancy. }\end{array}$ \\
\hline $\begin{array}{l}\text { Moussally et al., } \\
2009[36], \\
\text { Canada }\end{array}$ & Herbal & $\begin{array}{l}\text { Postnatal, } \\
3-8 \text { years } \\
\text { after birth }\end{array}$ & Questionnaire & $\begin{array}{l}3,354 / 8,505 \\
\quad(39.0)\end{array}$ & $\begin{array}{l}9 \% \text { used herbal medicines. } \\
\text { No data on stage of pregnancy. }\end{array}$ \\
\hline $\begin{array}{l}\text { Rahman et al., } \\
2009 \text { [37], } \\
\text { Malaysia }\end{array}$ & Herbal & Postnatal & Interview & 210 & $\begin{array}{l}52.4 \% \text { used herbal medicines. } \\
\text { Majority of use was in trimester } 3 \\
\text { (no } P \text { values). }\end{array}$ \\
\hline $\begin{array}{l}\text { Skouteris et al., } \\
2008[38] \\
\text { Australia }\end{array}$ & CAM & $\begin{array}{l}\text { Antenatal, } \\
\text { 24-31 weeks }\end{array}$ & Questionnaire & $\begin{array}{l}321 \text { ( } \mathrm{RR} \text { not } \\
\text { given) }\end{array}$ & $\begin{array}{l}73.2 \% \text { used at least one CAM, } \\
\text { mostly massage }(49.5 \%) \text {, and } \\
\text { vitamins/minerals }(49.5 \%) \text {. } \\
\text { No data on stage of pregnancy. }\end{array}$ \\
\hline
\end{tabular}

of the 22 studies linked CAM usage to pregnancy trimester $[18,19,23,25,26,29,31,34,37]$. Five of these nine studies $[18,19,26,29,37]$ reported increased use during the later stages of pregnancy, while three studies reported decreased use during pregnancy with the highest use during the first trimester [23, 25, 31]. Forster et al. reported insufficient data to draw any conclusions [34].

While 18 of the 22 studies [18, 20-28, 30-32, 34-38] quantified potential predictors of CAM use during pregnancy, only nine used a multivariate approach to analysis [20, 25, 28, 30, 32, 34-37]. Independent predictors for CAM use derived from these nine studies included CAM use prior to pregnancy $[20,28,35]$, higher educational attainment
[25, 26, 29], chronic disease/meds [28, 32, 36], ethnic background/nationality [21,28], higher income [21], and age [35].

A further major limitation of all 22 studies is the general lack of data on outcomes of perceived effectiveness, safety and overall satisfaction with treatment. While six studies included measures of effectiveness, none provided objective outcome data linked to specific CAM modalities, indications, and trimester of pregnancy. However, in these six studies, respondents' perceptions of effectiveness were generally positive, particularly in comparison with conventional approaches. Mohamed et al. reported that $56.2 \%$ of pregnant women thought that CAM was "more efficacious" and while $62.7 \%$ of pregnant women in the study by Lapi et al reported "equal 
TABLE 4: Data extraction relating to studies of professionals $(n=10)$.

\begin{tabular}{|c|c|c|c|c|c|}
\hline $\begin{array}{l}\text { Authors year, } \\
\text { country }\end{array}$ & $\begin{array}{l}\text { Professional } \\
\text { group }\end{array}$ & $\begin{array}{l}\text { CAMs studied } \\
\text { (authors' terms) }\end{array}$ & $\begin{array}{c}\text { Mode of data } \\
\text { collection }\end{array}$ & $\begin{array}{l}\text { Response rate } \\
(\mathrm{RR}, \%)\end{array}$ & $\begin{array}{l}\text { Prevalence } \\
\text { (\% recommending for patients) }\end{array}$ \\
\hline $\begin{array}{l}\text { Koc et al., } 2012 \\
\text { [39], Turkey }\end{array}$ & Midwives & CAM & Questionnaire & $129 / 159(81.1)$ & $\begin{array}{l}58.9 \% \text { used CAM in their practice, } \\
\text { mostly herbal medicines, diets, and } \\
\text { exercises }(32.6 \%, 27.9 \% \text {, and } 28.7 \%) \text {. }\end{array}$ \\
\hline $\begin{array}{l}\text { Dennehy et al., } \\
2010 \text { [40], USA }\end{array}$ & Midwives & $\begin{array}{l}\text { Herbal } \\
\text { medicines }\end{array}$ & Questionnaire & $\begin{array}{c}139 / 460 \\
(30.2)\end{array}$ & $\begin{array}{l}66.9 \% \text { used herbal medicines in } \\
\text { their practice. }\end{array}$ \\
\hline $\begin{array}{l}\text { Hrgovic et al., } 2010 \\
\text { [41], Croatia }\end{array}$ & $\begin{array}{l}\text { Heads of } \\
\text { obstetrics }\end{array}$ & CAM & Questionnaire & $36 / 36(100)$ & $\begin{array}{l}\text { Homeopathy, aromatherapy, } \\
\text { massage, moxibustion, } \\
\text { phytotherapy, acupressure, } \\
\text { reflexology, and Reiki were not used } \\
\text { at all (no data on herbal). } \\
\text { Acupuncture used at two centres } \\
\text { only. }\end{array}$ \\
\hline $\begin{array}{l}\text { Samuels et al., } 2010 \\
\text { [42], Israel }\end{array}$ & Midwives & CAM & Questionnaire & $173 / 238(72.7)$ & $\begin{array}{l}70 \% \text { used CAM in their practice } \\
\text { ( } 49.1 \% \text { massage, } 37.0 \% \text { herbals, and } \\
33.5 \% \text { homeopathy). }\end{array}$ \\
\hline $\begin{array}{l}\text { Harding and } \\
\text { Foureur, } 2009 \text { [43], } \\
\text { New Zealand, } \\
\text { Canada }\end{array}$ & Midwives & CAM & Questionnaire & $\begin{array}{c}343 / 648 \\
(52.9)\end{array}$ & $\begin{array}{l}\text { 71.95\% recommended or offered } \\
\text { CAM often ( } 31 \%) \text {, very frequently } \\
\text { ( } 28 \%) \text { or always (13\%). Most } \\
\text { common homeopathy, followed by } \\
\text { herbal medicines, aromatherapy, } \\
\text { and acupuncture (given as } \\
\text { frequencies for different patient } \\
\text { numbers, e.g., } 50 \% \text { of midwives } \\
\text { recommend for } 70-100 \% \text { of their } \\
\text { patients). }\end{array}$ \\
\hline $\begin{array}{l}\text { Hastings-Tolsm } \\
\text { and Terada, } 2009 \\
\text { [44], USA }\end{array}$ & Midwives & CAM & Questionnaire & $\begin{array}{l}227 / 500 \\
(45.0)\end{array}$ & $\begin{array}{l}78 \% \text { used CAM in their practice } \\
\text { mostly herbal medicines }(85 \%), \\
\text { pharmacologic/biologic treatments } \\
(82 \%) \text {, and mind-body } \\
\text { interventions }(80 \%) \text {. }\end{array}$ \\
\hline $\begin{array}{l}\text { Münstedt et al., } \\
2009 \text { [45], } \\
\text { Germany }\end{array}$ & $\begin{array}{l}\text { Heads of } \\
\text { obstetrics }\end{array}$ & CAM & Questionnaire & $138 / 187(73.4)$ & $\begin{array}{l}100 \% \text { offered acupuncture, } 95.7 \% \\
\text { homoeopathy, and } 50.7 \% \\
\text { aromatherapy. Decisions to provide } \\
\text { CAM were largely made by } \\
\text { midwives. }\end{array}$ \\
\hline $\begin{array}{l}\text { Münstedt et al., } \\
2009[46] \\
\text { Germany }\end{array}$ & $\begin{array}{c}\text { Head of } \\
\text { obstetrics }\end{array}$ & CAM & Questionnaire & $\begin{array}{c}381 / 946 \\
(40.3)\end{array}$ & $\begin{array}{l}97.3 \% \text { offered acupuncture, } 93.4 \% \\
\text { homeopathy, and } 76.6 \% \\
\text { aromatherapy. Decisions to provide } \\
\text { CAM were largely made by } \\
\text { midwives. }\end{array}$ \\
\hline $\begin{array}{l}\text { Wiebelitz et al., } \\
2009[47] \\
\text { Germany }\end{array}$ & $\begin{array}{l}\text { Midwives } \\
\text { (lecturers, } \\
\text { students) }\end{array}$ & CAM & Questionnaire & $309 / 309(100)$ & $\begin{array}{l}63.1 \% \text { estimated CAM to be } \\
\text { "applied frequently" (defined as } \\
>25 \% \text { of their pregnant patients) by } \\
\text { midwives. } 50-75 \% \text { estimated } \\
\text { homeopathy used "regularly", } \\
20-40 \% \text { phytotherapy, and } 28-27 \% \\
\text { hydrotherapy. }\end{array}$ \\
\hline $\begin{array}{l}\text { Furlow et al., } 2008 \\
\text { [48], USA }\end{array}$ & $\begin{array}{l}\text { Obstetric, } \\
\text { gynaecology } \\
\text { physicians }\end{array}$ & CAM & Questionnaire & $\begin{array}{l}401 / 1,009 \\
(41.0)\end{array}$ & $\begin{array}{l}97.6 \% \text { routinely endorsed, provided } \\
\text { or referred patients for at least one } \\
\text { CAM modality. } 86.4 \% \text { movement } \\
\text { therapies, } 80.3 \% \text { biofeedback etc. } \\
61.4 \% \text { herbal, and } 41.7 \% \\
\text { homeopathy. }\end{array}$ \\
\hline
\end{tabular}


effectiveness" $[28,31]$. In terms of herbals, Cuzzolin noted that $74.3 \%$ of respondents considered these to be "beneficial, reporting good results", with similar figures reported by Rahman et al. and Fakeye et al. Skouteris et al. commented that almost all (97.3\%) users of natural remedies "got completely better" or "got a bit better" [26, 33, 37, 38].

Only five studies assessed and reported the potential for possible CAM-related adverse effects (AE's) during pregnancy; however, both intra- and inter-study results were conflicting. While just over half of the respondents in two studies [28, 31] viewed CAM approaches to be safer than traditional medicine, the majority had concerns about the safety of herbals. Kochhar et al. reported that $67.7 \%$ of respondents agreed that herbals could harm a baby if taken during pregnancy, and Bercaw et al. reported that a minority of respondents $(22 \%)$ agreed that herbals were safer to use than prescribed medicines [24, 27]. Similarly, Fakeye et al. reported that $33.4 \%$ of respondents believed that herbals possessed no AEs [33]. Although three of these studies did report observed AEs, none provided precise details of the method used for AE identification (specific tick list or open comments). Fakeye et al. reported that $18 \%$ of those taking herbals had "some form of unwanted effects" such as vomiting, and dizziness. Cuzzolin and Benoni and Leppee et al. both reported that $3.7 \%$ of respondents experienced mild AEs [26, 29, 33].

Only one study (Kalder et al.) reported on overall user satisfaction with treatment. The authors limited their report to that "almost all respondents were satisfied" [20].

3.2. Studies Assessing Experience and Views of Healthcare Professionals $(n=10)$. Of the ten studies, nine reported data on more than one CAM modality and one on herbals only.

3.2.1. Definitions and Scope. Of the nine studies reporting data for more than one CAM modality, only four [39, 42, 43,48 ] defined "CAM" while eight provided a detailed and specific checklist of CAM modalities and products. Dennehy et al. focused on herbals but neither defined "herbals" nor described a specific checklist [40].

3.2.2. Study Design. Questionnaires were used in all ten studies, with sample sizes varying from 36 (heads of obstetric departments in Croatia) [41] to 1,009 (obstetric gynaecology physicians in USA) [48]. All ten studies used a convenience approach to participant sampling and recruitment with subsequent limitation of external validity of findings. Response rates ranged from $30.2 \%$ [40] to $100 \%$ [41, 47]; all those with response rates less than $100 \%$ did not consider homogeneity of respondents and nonrespondents.

3.2.3. Study Findings. Six studies [39, 40, 42-44, 47] assessed the views and experiences of midwives while the remaining four studies focused on obstetricians [41, 45, 46, 48]. Despite limitations of study design and reporting, most respondents (other than heads of obstetric departments in Germany) [41] used CAMs in their practice.

Issues of perceived effectiveness or safety of CAMs were quantified in two studies. Koc et al. reported that $61.2 \%$ of midwife respondents in Turkey thought that CAM would be "beneficial", $24.9 \%$ that CAM use decreased pregnancyrelated complaints, and $61.2 \%$ that CAM use may have AEs [39]. In a study of obstetric physicians in USA, Furlow et al. reported that more than $50 \%$ of respondents had positive beliefs on the effectiveness of biofeedback, chiropractic, acupuncture, and meditation whereas $41.2 \%$ and $24.9 \%$ had positive views on herbals and homeopathic preparations, respectively [48]. Importantly, while the study by Harding and Foureur did not report objective measures of effectiveness or safety of CAM, this study did report that $71.0 \%$ of midwife respondents in the USA viewed CAM to be an "essential part of midwifery practice" [43].

\section{Discussion}

Despite a lack of evidence for efficacy and safety, CAM use is reportedly increasing worldwide. Of possible concern is the use of CAM modalities, such as herbals, in high risk patient groups such as pregnant women. The results of this systematic review of the recent published literature highlight the high levels of systemic CAM use, by women and healthcare professionals, during pregnancy.

However, it is clear that the majority of studies assessing CAM use during pregnancy have limitations in terms of both study design and reporting. Specific weaknesses were a lack of appropriate definitions of CAM and associated modalities, the absence of detailed checklists provided to research participants, the frequent use of convenience sampling, and limited detail of considerations of face and content of questionnaire items and test-retest reliability. For these reasons, it was not possible to pool the data from identified studies in an appropriate way to generate meaningful and generalisable conclusions.

Our systematic review was conducted according to best practice as defined by the Centre for Reviews and Dissemination [13]. Of note each paper was independently reviewed by two authors using standard evidence-based critical appraisal criteria [14]. To ensure that this review was current we are restricted to peer reviewed reports of primary data published during the last five years.

The application of a consistent and useable CAM definition proved to be a major issue. While CAM has been defined by the WHO [1], this definition is vague and open to multiple interpretations, particularly interpretation of "tradition" and "healthcare systems". Indeed, whether or not a particular therapy is deemed to be CAM may differ between countries, healthcare settings, and specialities. This situation could readily be overcome by the use of standardised, validated checklists. In those studies where checklists were issued to participants, the list of CAM products and modalities included varied widely leading to potentially significant differences in participant product identification. Without a clear and specific definition of each CAM modality and CAM products, it is impossible to perform study comparison or pool data for the purposes of meta-analysis. The lack of definitions and checklists may explain why the reported prevalence of CAM use appears to be highly variable, even within similar populations. 
Furthermore, the majority of studies reviewed employed a retrospective method of data collection, with the subsequent limitation of recall bias. The ideal study design which in the case of pregnant women should be prospective and longitudinal, collecting data both before and throughout the course of pregnancy and preferably following delivery should be given consideration in future studies. This would reduce the inherent effects of recall bias and furthermore would permit linkage of CAM use with indication, trimester of pregnancy, and outcome.

Most studies relied on single centre data collection using a convenience approach to sampling. While this may be justified in terms of study logistics, there are clear implications for external validity. Furthermore, a sample size calculation was rarely reported and presenting lacked full statistical justification, thus having implications for confidence in reported outcomes.

While inferential statistics were employed, there was often a lack of multivariate approaches, which may be one of the reasons for the conflicting results and conclusions in relation to factors influencing the use of CAMs during pregnancy.

There is a need to expand the evidence base assessing CAMs use during pregnancy in terms of prevalence with emphasis on outcomes of effectiveness and potential AEs. Efficacy studies are required for those modalities and products widely used by women during pregnancy and those recommended by healthcare professionals. In addition, qualitative exploration of the reasons for use, factors affecting use, and position of CAMs within the overall treatment hierarchy is warranted.

\section{Study Highlights}

(i) Reportedly CAM's are widely used by women during pregnancy.

(ii) What is the quality of recent published evidence reporting the views, experiences of pregnant women and healthcare professionals towards CAM use during pregnancy?

(iii) The quality of the published literature is limited in terms of both study design and reporting.

(iv) There is an urgent need to expand the research based on CAM use in pregnancy. Appropriately designed efficacy studies are required for those CAM modalities and products widely used.

\section{Conflict of Interests}

The authors do not have any issues of involvement, financial, conflict or otherwise that might potentially bias his or her work.

\section{Authors' Contribution}

James S. McLay, Derek Stewart, Abdul Rouf Pallivalappila, Ashalatha Shetty, and Binita Pande designed the review. All authors contributed to phases of data collection, synthesis, writing, and reviewing the paper.

\section{References}

[1] World Health Organisation, Traditional Medicines: Definitions, 2013, http://www.who.int/medicines/areas/traditional/definitions/en/index.html.

[2] E. Ernst, M. H. Pittler, and B. Wider, The Desktop Guide to Complementary and Alternative Medicine, Mosby Elsevier, Amsterdam, The Netherlands, 2006.

[3] K. J. Hunt, H. F. Coelho, B. Wider et al., "Complementary and alternative medicine use in England: results from a national survey," International Journal of Clinical Practice, vol. 64, no. 11, pp. 1496-1502, 2010.

[4] J. Sarris, T. B. R. Wahlin, D. C. Goncalves, and G. J. Byrne, "Comparative use of complementary medicine, allied health, and manual therapies by middle-aged and older Australian women," Journal of Women \& Aging, vol. 22, no. 4, pp. 273-282, 2010.

[5] C. A. Lunny and S. N. Fraser, "The use of complementary and alternative medicines among a sample of Canadian menopausal-aged women," Journal of Midwifery \& Women's Health, vol. 55, no. 4, pp. 335-343, 2010.

[6] C. S. Broussard, C. Louik, M. A. Honein, and A. A. Mitchell, "Herbal use before and during pregnancy," American Journal of Obstetrics \& Gynecology, vol. 202, no. 5, pp. 443.el-443.e6, 2010.

[7] A. Fugh-Berman and F. Kronenberg, "Complementary and alternative medicine (CAM) in reproductive-age women: a review of randomized controlled trials," Reproductive Toxicology, vol. 17, no. 2, pp. 137-152, 2003.

[8] D. M. Marcus and W. R. Snodgrass, "Do no harm: avoidance of herbal medicines during pregnancy," Obstetrics \& Gynecology, vol. 105, no. 5, part 1, pp. 1119-1122, 2005.

[9] J. Adams, D. Sibbritt, A. Broom et al., "Women's use of complementary and alternative medicine during pregnancy: a critical review of the literature," Birth, vol. 36, no. 3, pp. 237-245, 2009.

[10] H. G. Hall, D. L. Griffiths, and L. G. McKenna, "The use of complementary and alternative medicine by pregnant women: a literature review," Midwifery, vol. 27, no. 6, pp. 817-824, 2011.

[11] A. Steel, J. Adams, and D. Sibbritt, "Complementary and alternative medicine in pregnancy: a systematic review," Journal of the Australian Traditional-Medicine Society, vol. 17, no. 4, p. 205, 2012.

[12] H. G. Hall, L. G. McKenna, and D. L. Griffiths, "Midwives' support for complementary and alternative medicine: a literature review," Women and Birth, vol. 25, no. 1, pp. 4-12, 2012.

[13] Systematic Reviews: CRD's guidance for undertaking reviews in health care, 2009, http://www.york.ac.uk/inst/crd/SysRev/ !SSL!/WebHelp/SysRev3.htm.

[14] Scottish Intercollegiate Guidelines Network. Search filters, 2011, http://www.sign.ac.uk/methodology/filters.html.

[15] J. P. Vandenbroucke, E. von Elm, D. G. Altman et al., "Strengthening the reporting of observational studies in epidemiology (STROBE): explanation and elaboration," Gaceta Sanitaria, vol. 23, no. 2, pp. 158.e1-158.e28, 2009.

[16] F. J. Fowler Jr., Survey Research Methods, vol. 1, Sage Publications, 2008.

[17] J. Adams, D. Sibbritt, and C. W. Lui, "The use of complementary and alternative medicine during pregnancy: a longitudinal study of Australian women," Birth, vol. 38, no. 3, pp. 200-206, 2011.

[18] J. L. Bishop, K. Northstone, J. R. Green, and E. A. Thompson, "The use of complementary and alternative medicine in pregnancy: data from the Avon Longitudinal Study of Parents and 
Children (ALSPAC)," Complementary Therapies in Medicine, vol. 19, no. 6, pp. 303-310, 2011.

[19] I. M. Al-Riyami, I. Q. Al-Busaidy, and I. S. Al-Zakwani, "Medication use during pregnancy in Omani women," International Journal of Clinical Pharmacy, vol. 33, no. 4, pp. 634-641, 2011.

[20] M. Kalder, K. Knoblauch, I. Hrgovic, and K. Münstedt, "Use of complementary and alternative medicine during pregnancy and delivery," Archives of Gynecology and Obstetrics, vol. 283, no. 3, pp. 475-482, 2011.

[21] R. Khresheh, "How women manage nausea and vomiting during pregnancy: a Jordanian study," Midwifery, vol. 27, no. 1, pp. 42-45, 2011.

[22] H. Nordeng, K. Bayne, G. C. Havnen, and B. S. Paulsen, "Use of herbal drugs during pregnancy among 600 Norwegian women in relation to concurrent use of conventional drugs and pregnancy outcome," Complementary Therapies in Clinical Practice, vol. 17, no. 3, pp. 147-151, 2011.

[23] M. Tabatabaee, "Use of herbal medicine among pregnant women referring to Valiasr hospital in Kazeroon, Fars, South of Iran," Journal of Medicinal Plants, vol. 10, no. 37, pp. 96-108, 2011.

[24] J. Bercaw, B. Maheshwari, and H. Sangi-Haghpeykar, "The use during pregnancy of prescription, over-the-counter, and alternative medications among Hispanic women," Birth, vol. 37, no. 3, pp. 211-218, 2010.

[25] C. S. Broussard, C. Louik, M. A. Honein et al., "Herbal use before and during pregnancy," American Journal of Obstetrics \& Gynecology, vol. 202, no. 5, pp. 443.el-443.e6, 2010.

[26] L. Cuzzolin and G. Benoni, "Safety issues of phytomedicines in pregnancy and paediatrics," in Herbal Drugs: Ethnomedicine to Modern Medicine, K. G. Ramawat, Ed., pp. 381-396, Springer, Berlin, Germany, 2009.

[27] K. Kochhar, R. M. Saywell Jr., T. W. Zollinger et al., "Herbal remedy use among hispanic women during pregnancy and while breastfeeding: are physicians informed?" Hispanic Health Care International, vol. 8, no. 2, pp. 93-106, 2010.

[28] F. Lapi, A. Vannacci, M. Moschini et al., "Use, attitudes and knowledge of complementary and alternative drugs (CADs) among pregnant women: a preliminary survey in tuscany," Evidence-Based Complementary and Alternative Medicine, vol. 7, no. 4, pp. 477-486, 2010.

[29] M. Leppee, J. Culig, M. Eric, J. Boskovic, and N. Colak, "Vitamin, mineral and iron supplementation in pregnancy: crosssectional study," Biopolymers and Cell, vol. 26, no. 2, pp. 128-135, 2010.

[30] C. Louik, P. Gardiner, K. Kelley, and A. A. Mitchell, "Use of herbal treatments in pregnancy," American Journal of Obstetrics \& Gynecology, vol. 202, no. 5, pp. 439.el-439.e10, 2010.

[31] H. Mohamed, J. Abdin, and D. Al Kozaai, "Knowledge, attitude and practice of complementary and alternative medicine (CAM) among pregnant women: a preliminary survey in Qatar," Middle East Journal of Family Medicine, vol. 7, no. 10, pp. 5-14, 2010.

[32] C. H. Chuang, P. J. Chang, W. S. Hsieh et al., "Chinese herbal medicine use in Taiwan during pregnancy and the postpartum period: a population-based cohort study," International Journal of Nursing Studies, vol. 46, no. 6, pp. 787-795, 2009.

[33] T. O. Fakeye, R. Adisa, and I. E. Musa, "Attitude and use of herbal medicines among pregnant women in Nigeria," BMC Complementary and Alternative Medicine, vol. 9, article 53, 2009.
[34] D. A. Forster, G. Wills, A. Denning, and M. Bolger, “The use of folic acid and other vitamins before and during pregnancy in a group of women in Melbourne, Australia," Midwifery, vol. 25, no. 2, pp. 134-146, 2009.

[35] L. Holst, D. Wright, H. Nordeng, and S. Haavik, "Use of herbal preparations during pregnancy: focus group discussion among expectant mothers attending a hospital antenatal clinic in Norwich, UK," Complementary Therapies in Clinical Practice, vol. 15, no. 4, pp. 225-229, 2009.

[36] K. Moussally, D. Oraichi, and A. Bérard, "Herbal products use during pregnancy: prevalence and predictors," Pharmacoepidemiology and Drug Safety, vol. 18, no. 6, pp. 454-461, 2009.

[37] A. A. Rahman, S. A. Sulaiman, Z. Ahmad, H. Salleh, W. N. W. Daud, and A. M. Hamid, "Women's attitude and sociodemographic characteristics influencing usage of herbal medicines during pregnancy in Tumpat District, Kelantan," Southeast Asian Journal of Tropical Medicine and Public Health, vol. 40, no. 2, pp. 330-337, 2009.

[38] H. Skouteris, E. H. Wertheim, S. Rallis et al., "Use of complementary and alternative medicines by a sample of Australian women during pregnancy," Australian and New Zealand Journal of Obstetrics and Gynaecology, vol. 48, no. 4, pp. 384-390, 2008.

[39] Z. Koc, S. Topatan, and Z. Saglam, "Use of and attitudes toward complementary and alternative medicine among midwives in Turkey," European Journal of Obstetrics Gynecology and Reproductive Biology, vol. 160, no. 2, pp. 131-136, 2012.

[40] C. Dennehy, C. Tsourounis, L. Bui, and T. L. King, "The use of herbs by California Midwives," Journal of Obstetric, Gynecologic, and Neonatal Nursing, vol. 39, no. 6, pp. 684-693, 2010.

[41] I. Hrgovic, Z. Hrgovic, D. Habek, S. Oreskovic, J. Hofmann, and K. Münstedt, "Use of complementary and alternative medicine in departments of obstetrics in Croatia and a comparison to Germany," Forschende Komplementarmedizin, vol. 17, no. 3, pp. 144-146, 2010.

[42] N. Samuels, R. Y. Zisk-Rony, S. R. Singer et al., "Use of and attitudes toward complementary and alternative medicine among nurse-midwives in Israel," American Journal of Obstetrics \& Gynecology, vol. 203, no. 4, pp. 341.el-341.e7, 2010.

[43] D. Harding and M. Foureur, "New Zealand and Canadian midwives' use of complementary and alternative medicine," New Zealand College of Midwives Journal, vol. 40, pp. 7-12, 2009.

[44] M. Hastings-Tolsma and M. Terada, "Complementary medicine use by nurse midwives in the U.S," Complementary Therapies in Clinical Practice, vol. 15, no. 4, pp. 212-219, 2009.

[45] K. Münstedt, C. Schröter, D. Brüggmann, H. R. Tinneberg, and R. von Georgi, "Use of complementary and alternative medicine in departments of obstetrics in Germany," Forschende Komplementarmedizin, vol. 16, no. 2, pp. 111-116, 2009.

[46] K. Münstedt, A. Brenken, and M. Kalder, "Clinical indications and perceived effectiveness of complementary and alternative medicine in departments of obstetrics in Germany: a questionnaire study," European Journal of Obstetrics Gynecology and Reproductive Biology, vol. 146, no. 1, pp. 50-54, 2009.

[47] K. R. Wiebelitz, T. W. Goecke, J. Brach, and A. M. Beer, "Use of complementary and alternative medicine in obstetrics," British Journal of Midwifery, vol. 17, no. 3, pp. 169-175, 2009.

[48] M. L. Furlow, D. A. Patel, A. Sen, and J. R. Liu, "Physician and patient attitudes towards complementary and alternative medicine in obstetrics and gynecology," BMC Complementary and Alternative Medicine, vol. 8, article 35, 2008. 


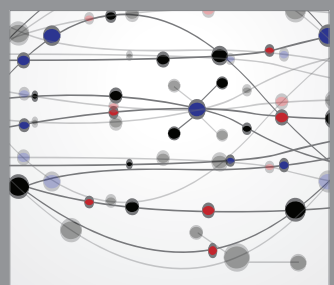

The Scientific World Journal
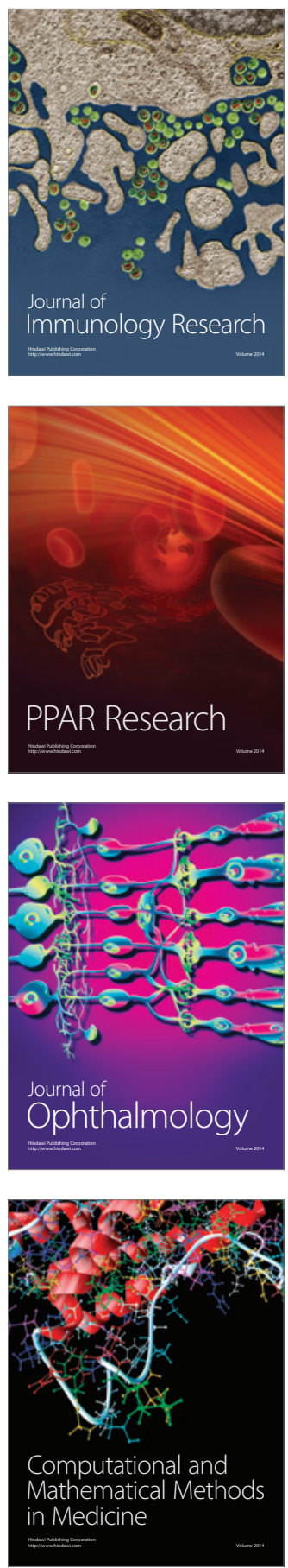

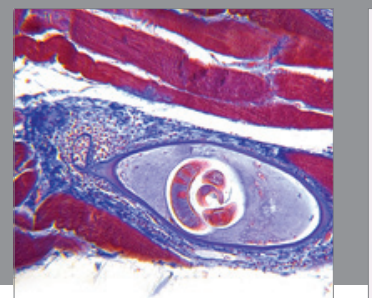

Gastroenterology

Research and Practice
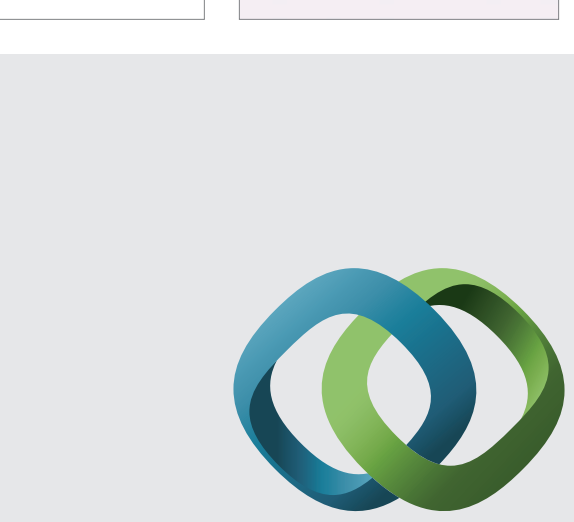

\section{Hindawi}

Submit your manuscripts at

http://www.hindawi.com
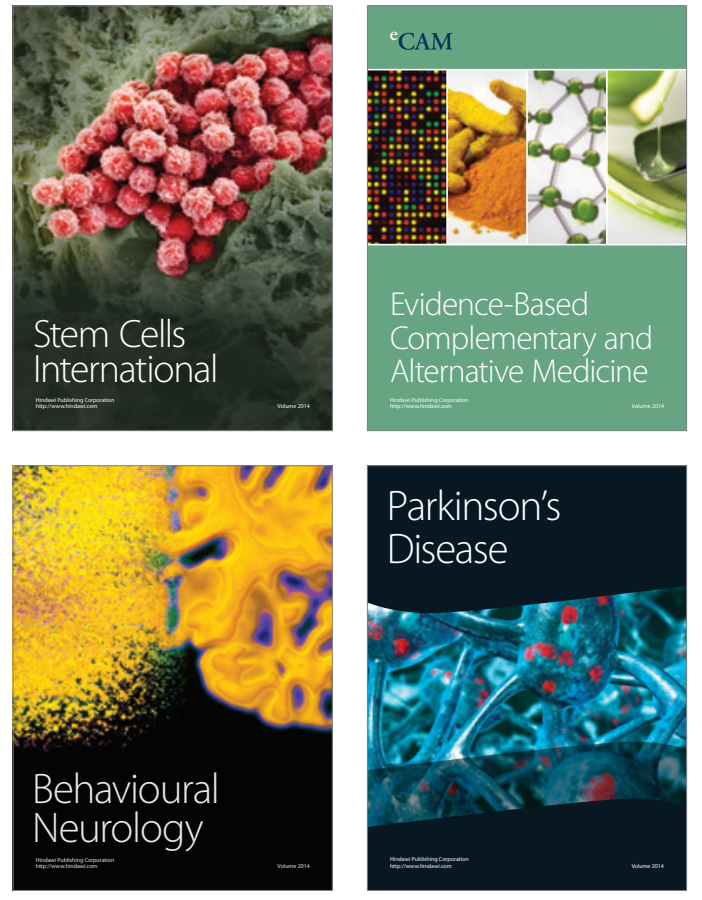
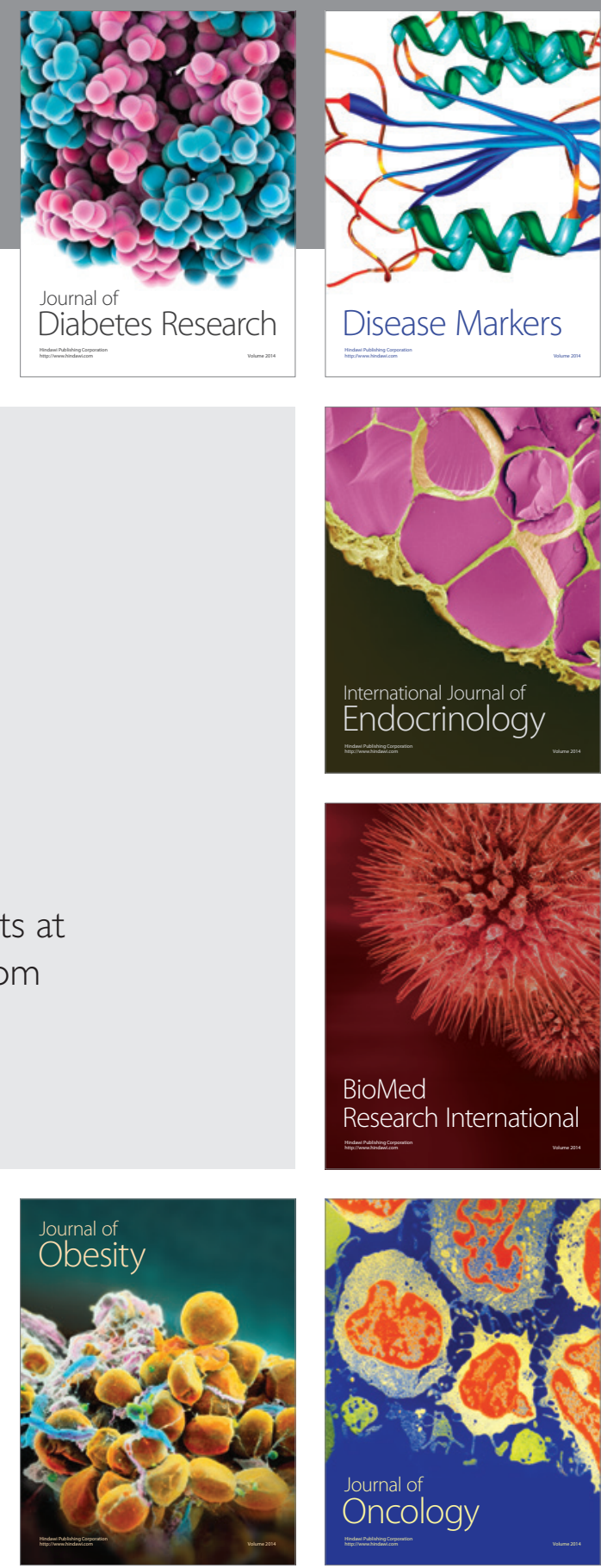

Disease Markers
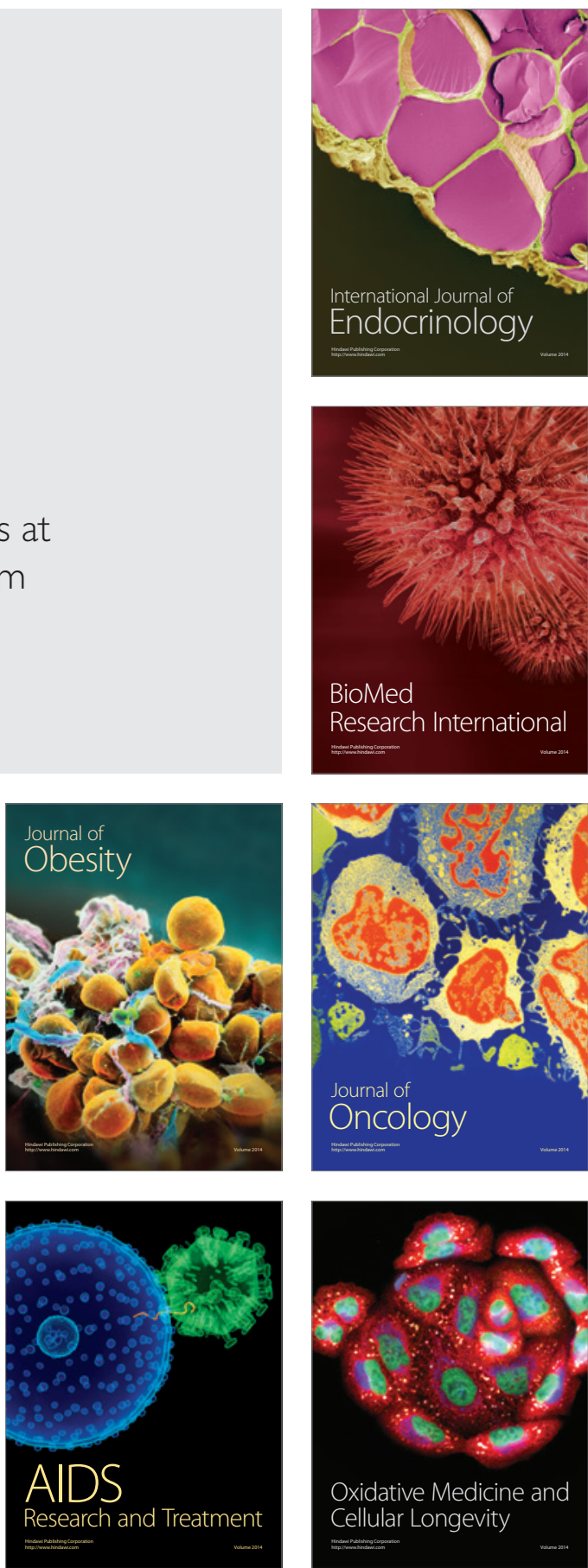